\title{
Crucial abelian k-power-free words
}

\author{
Amy Glen 诲 Bjarni V. Halldórsson啡 Sergey Kitaev非 \\ ${ }^{1}$ Department of Mathematics and Statistics, School of Chemical and Mathematical Sciences, Murdoch University, \\ Perth, WA 6150, AUSTRALIA \\ ${ }^{2}$ Reykjavík University, Menntavegur 1, 101 Reykjavík, ICELAND
}

received $6^{\text {th }}$ July 2009, revised $31^{\text {st }}$ October 2010, accepted $19^{\text {th }}$ November 2010.

In 1961, Erdős asked whether or not there exist words of arbitrary length over a fixed finite alphabet that avoid patterns of the form $X X^{\prime}$ where $X^{\prime}$ is a permutation of $X$ (called abelian squares). This problem has since been solved in the affirmative in a series of papers from 1968 to 1992 . Much less is known in the case of abelian $k$-th powers, i.e., words of the form $X_{1} X_{2} \cdots X_{k}$ where $X_{i}$ is a permutation of $X_{1}$ for $2 \leq i \leq k$.

In this paper, we consider crucial words for abelian $k$-th powers, i.e., finite words that avoid abelian $k$-th powers, but which cannot be extended to the right by any letter of their own alphabets without creating an abelian $k$-th power. More specifically, we consider the problem of determining the minimal length of a crucial word avoiding abelian $k$-th powers. This problem has already been solved for abelian squares by Evdokimov and Kitaev (2004), who showed that a minimal crucial word over an $n$-letter alphabet $\mathcal{A}_{n}=\{1,2, \ldots, n\}$ avoiding abelian squares has length $4 n-7$ for $n \geq 3$. Extending this result, we prove that a minimal crucial word over $\mathcal{A}_{n}$ avoiding abelian cubes has length $9 n-13$ for $n \geq 5$, and it has length 2, 5, 11, and 20 for $n=1,2,3$, and 4, respectively. Moreover, for $n \geq 4$ and $k \geq 2$, we give a construction of length $k^{2}(n-1)-k-1$ of a crucial word over $\mathcal{A}_{n}$ avoiding abelian $k$-th powers. This construction gives the minimal length for $k=2$ and $k=3$. For $k \geq 4$ and $n \geq 5$, we provide a lower bound for the length of crucial words over $\mathcal{A}_{n}$ avoiding abelian $k$-th powers.

MSC (2010): 05D99; 68R05; 68R 15

Keywords: pattern avoidance; abelian square-free word; abelian cube-free word; abelian power; crucial word; Zimin word

\section{Introduction}

Let $\mathcal{A}_{n}=\{1,2, \ldots, n\}$ be an $n$-letter alphabet and let $k \geq 2$ be an integer. A word $W$ over $\mathcal{A}_{n}$ contains a $k$-th power if $W$ has a factor of the form $X^{k}=X X \cdots X$ ( $k$ times) for some non-empty word $X$. A $k$-th power is trivial if $X$ is a single letter. For example, the word $V=13243232323243$ over $\mathcal{A}_{4}$ contains the (non-trivial) 4 -th power $(32)^{4}=32323232$. A word $W$ contains an abelian $k$-th power if $W$ has a factor of the form $X_{1} X_{2} \cdots X_{k}$ where $X_{i}$ is a permutation of $X_{1}$ for $2 \leq i \leq k$. The cases $k=2$ and $k=3$

\footnotetext{
†Email: amy.glen@gmail.com

¥Email: bjarnivh@ru. is

$\S$ Email:sergey@ru.is
}

1365-8050 @ 2010 Discrete Mathematics and Theoretical Computer Science (DMTCS), Nancy, France 
give us (abelian) squares and cubes, respectively. For instance, the preceding word $V$ contains the abelian square 4323232324 and the word 123312213 is an abelian cube. A word $W$ is (abelian) $k$-power-free if $W$ avoids (abelian) $k$-th powers, i.e., if $W$ does not contain any (abelian) $k$-th powers. For example, the word 1234324 is abelian cube-free, but not abelian square-free since it contains the abelian square 234324 .

A word $W$ is crucial with respect to a given set of prohibited words (or simply prohibitions) if $W$ avoids the prohibitions, but $W x$ does not avoid the prohibitions for any letter $x$ occurring in $W$. A minimal crucial word is a crucial word of the shortest length. For example, the word $W=21211$ (of length 5) is crucial with respect to abelian cubes since it is abelian cube-free and the words $W 1$ and $W 2$ end with the abelian cubes 111 and 212112 , respectively. Actually, $W$ is a minimal crucial word over $\{1,2\}$ with respect to abelian cubes. Indeed, one can easily verify that there do not exist any crucial abelian cube-free words on two letters of length less than 5 .

Abelian squares were first introduced by Erdôs [4], who asked whether or not there exist words of arbitrary length over a fixed finite alphabet that avoid patterns of the form $X X^{\prime}$ where $X^{\prime}$ is a permutation of $X$ (i.e., abelian squares). This question has since been solved in the affirmative in a series of papers from 1968 to 1992 (see [5, 9, 7] and also [2]). Problems of this type were also considered by Zimin [10], who used the following sequence of words as a key tool.

The Zimin word $Z_{n}$ over $\mathcal{A}_{n}$ is defined recursively as follows: $Z_{1}=1$ and $Z_{n}=Z_{n-1} n Z_{n-1}$ for $n \geq 2$. The first four Zimin words are:

$$
Z_{1}=1, Z_{2}=121, Z_{3}=1213121, Z_{4}=121312141213121 .
$$

The $k$-generalized Zimin word $Z_{n, k}=X_{n}$ is defined as

$$
X_{1}=1^{k-1}=11 \cdots 1, X_{n}=\left(X_{n-1} n\right)^{k-1} X_{n-1}=X_{n-1} n X_{n-1} n \cdots n X_{n-1}
$$

where the number of 1's, as well as the number of $n$ 's, is $k-1$. Thus $Z_{n}=Z_{n, 2}$. It is easy to see (by induction) that each $Z_{n, k}$ avoids (abelian) $k$-th powers and has length $k^{n}-1$. Moreover, it is known that $Z_{n, k}$ gives the length of a minimal crucial word avoiding $k$-th powers.

However, much less is known in the case of abelian powers. Crucial abelian square-free words (also called right maximal abelian square-free words) of exponential length are given in [3] and [6], and it is shown in [6] that a minimal crucial abelian square-free word over an $n$-letter alphabet has length $4 n-7$ for $n \geq 3$.

In this paper, we extend the study of crucial abelian $k$-power-free words to the case of $k>2$. In particular, we provide a complete solution to the problem of determining the length of a minimal crucial abelian cube-free word (the case $k=3$ ) and we conjecture a solution in the general case. More precisely, we show that a minimal crucial word over $\mathcal{A}_{n}$ avoiding abelian cubes has length $9 n-13$ for $n \geq 5$ (Corollary 9), and it has length 2, 5, 11, and 20 for $n=1,2,3$, and 4, respectively. For $n \geq 4$ and $k \geq 2$, we give a construction of length $k^{2}(n-1)-k-1$ of a crucial word over $\mathcal{A}_{n}$ avoiding abelian $k$-th powers (see Theorem 11). This construction gives the minimal length for $k=2$ and $k=3$, and we conjecture that this is also true for any $k \geq 4$ and sufficiently large $n$. We also provide a rough lower bound for the length of minimal crucial words over $\mathcal{A}_{n}$ avoiding abelian $k$-th powers, for $n \geq 5$ and $k \geq 4$ (see Theorem 12.

For a crucial word $X$ over $\mathcal{A}_{n}$, we let $X=X_{i} \Delta_{i}$ where $\Delta_{i}$ is the factor of minimal length such that $\Delta_{i} i$ is a prohibition for $i \in \mathcal{A}_{n}$. Note that we can rename letters, if needed, so we can assume that for any 
minimal crucial word $X$, one has

$$
\Delta_{1} \subset \Delta_{2} \subset \cdots \subset \Delta_{n}=X
$$

where " $\subset$ " means (proper) right factor (or suffix). In other words, for each $i=2,3, \ldots, n$, we have $\Delta_{i}=Y_{i} \Delta_{i-1}$ for some non-empty $Y_{i}$. In what follows we will use $X_{i}$ and $Y_{i}$ as stated above. We note that the definitions imply:

$$
X=X_{i} \Delta_{i}=X_{i} Y_{i} \Delta_{i-1}=X_{n-1} Y_{n-1} Y_{n-2} \cdots Y_{2} \Delta_{1}
$$

for any $i=2,3, \ldots, n-1$. Furthermore, in the case of crucial words avoiding abelian $k$-th powers, we write $\Delta_{i} i=\Omega_{i, 1} \Omega_{i, 2} \cdots \Omega_{i, k}$, where the $k$ blocks $\Omega_{i, j}$ are equal up to permutation, and we denote by $\Omega_{i, k}^{\prime}$ the block $\Omega_{i, k}$ without the rightmost $i$.

Hereafter, we let $\ell_{k}(n)$ denote the length of a minimal crucial word over $\mathcal{A}_{n}$ avoiding abelian $k$-th powers. The length of a word $W$ is denoted by $|W|$, and we denote by $|W|_{x}$ the number of occurrences of a letter $x$ in $W$. The Parikh vector of a word $W$ over $\mathcal{A}_{n}$ is defined by

$$
\mathcal{P}(W):=\left(|W|_{1},|W|_{2}, \ldots,|W|_{n}\right) .
$$

Clearly, if $W$ is an abelian $k$-th power, then $|W|_{x} \equiv 0(\bmod k)$ for all letters $x$ occurring in $W$.

\section{Crucial words for abelian cubes}

\subsection{An upper bound for $\ell_{3}(n)$}

The fact that the 3-generalized Zimin word $Z_{n, 3}$ is crucial with respect to abelian cubes already gives us an upper bound of $3^{n}-1$ for $\ell_{3}(n)$. In Theorem 1 (below) we improve this upper bound to $3 \cdot 2^{n-1}-1$. We then discuss a further improvement of the bound using a greedy algorithm, which gives asymptotically $O\left((\sqrt{3})^{n}\right)$. This greedy algorithm provides minimal crucial abelian cube-free words over $\mathcal{A}_{n}$ for $n=3$ and $n=4$, while the construction in Theorem 1 is optimal only for $n=3$. We also provide a construction of a crucial word of length $9 n-10$ which exceeds our lower bound by only 3 letters, for $n \geq 5$. Finally, we end this section with a construction of a crucial abelian cube-free word over $\mathcal{A}_{n}$ of length $9 n-13$, which coincides with the lower bound given in Theorem 8 of Section 2.2 for $n \geq 5$.

Theorem 1 One has that $\ell_{3}(n) \leq 3 \cdot 2^{n-1}-1$.

Proof: We construct a crucial abelian cube-free word $X=X_{n}$ iteratively as follows. Set $X_{1}=11$ and assume $X_{n-1}$ has been constructed. Then do the following:

1. Increase all letters of $X_{n-1}$ by 1 to obtain $X_{n-1}^{\prime}$.

2. Insert 1 after (to the right of) each letter of $X_{n-1}^{\prime}$ and adjoin one extra 1 to the right of the resulting word to get $X_{n}$.

For example, $X_{2}=21211, X_{3}=31213121211$, etc. It is easy to verify that $\left|X_{n}\right|=3 \cdot 2^{n-1}-1$. We show by induction that $X_{n}$ avoids abelian cubes, whereas $X_{n} x$ does not avoid abelian cubes for any $x \in \mathcal{A}_{n}$. Both claims are trivially true for $n=1$. Now take $n \geq 2$. If $X_{n}$ contains an abelian cube, then removing all 1's from it, we would deduce that $X_{n-1}$ must also contain an abelian cube, contradicting the fact that $X_{n-1}$ contains no abelian cubes. 
It remains to show that extending $X_{n}$ to the right by any letter $x$ from $\mathcal{A}_{n}$ creates an abelian cube. If $x=1$ then we get 111 from the construction of $X_{n}$. On the other hand, if $x>1$ then we swap the rightmost 1 with the rightmost $x$ in $X x$, thus obtaining a word where every other letter is 1 ; removing all 1 's and decreasing each of the remaining letters by 1 , we have $X_{n-1}(x-1)$, which contains an abelian cube (by the induction hypothesis).

As a further improvement of Theorem 1 we sketch here, without providing all the details, the work of a greedy algorithm. Here, "greedy" means that we assume (by induction) that an "optimal" crucial abelian cube-free word $X_{n-1}$ over $\mathcal{A}_{n-1}$ has been constructed. Next (for the greedy step) we add just two $n$ 's (the minimum possible), and then we add as few of the other letters as possible to build a crucial abelian cubefree word $X_{n}$ over $\mathcal{A}_{n}$. More precisely, we set $\Delta_{1}=11$ and assuming that $\Delta_{i-1}$ is built, we consider the minimum list $T_{i}$ of letters we must add (forming $Y_{i}$ and possibly updating $\Delta_{i-1}$ by permuting letters in $\left.Y_{i-1}\right)$ to build $\Delta_{i}$, for $i=2,3, \ldots, n$, which can then be turned into a construction of a crucial abelian cube-free word $X_{n}$ over $\mathcal{A}_{n}$. It is easy to see from the definitions of an abelian cube and a crucial word that $T_{2}$ must contain at least two 2's and at least one 1. Then $T_{3}$ must contain at least two 3's, at least one 2, and at least three 1's (the last statement follows from the fact that the two 3's in $\Delta_{3}$ are supposed to be accompanied by at least one 1 in $\Omega_{3,1}$ and $\Omega_{3,2}$ in $\Delta_{3} 3=\Omega_{3,1} \Omega_{3,2} \Omega_{3,3}$, but without extra 1's, whose number of occurrences must be divisible by 3 , we cannot manage it). Running this type of argument, one can come up with the following $T_{i}$ for initial values of $i$ :

$$
\begin{gathered}
T_{2}=\{1,2,2\}, T_{3}=\{1,1,1,2,3,3\}, T_{4}=\{1,1,1,2,2,2,3,4,4\}, \\
T_{5}=\{1,1,1,1,1,1,1,1,1,2,2,2,3,3,3,4,5,5\}, \\
T_{6}=\{1,1,1,1,1,1,1,1,1,2,2,2,2,2,2,2,2,2,3,3,3,4,4,4,5,6,6\}, \text { etc. }
\end{gathered}
$$

In particular, we observe that $\left(\left|T_{n}\right|\right)_{n \geq 1}=2,3,6,9,18,27, \ldots$ where

$$
\left|T_{2 i+1}\right|=2 \cdot 3^{i} \quad \text { and } \quad\left|T_{2 i+2}\right|=3^{i} \quad \text { for all } i \geq 0 .
$$

Hence, these considerations lead to a crucial abelian cube-free word over an $n$-letter alphabet of length

$$
\sum_{j=0}^{\left\lfloor\frac{n-1}{2}\right\rfloor} 2 \cdot 3^{j}+\sum_{j=1}^{\left\lfloor\frac{n}{2}\right\rfloor} 3^{j} .
$$

Initial values for the lengths are $2,5,11,20,38,65, \ldots$ Furthermore, by observing that $\sum_{j=0}^{m} 3^{j}=$ $\frac{3^{m+1}-1}{2}$ for any integer $m \geq 0$ (which can be easily proved by induction), we deduce that the length of a crucial abelian cube-free word obtained in this way is asymptotically $O\left((\sqrt{3})^{n}\right)$. Even though this length is exponential, we $d o$ in fact obtain optimal values for $n \leq 4$. Below we list optimal abelian cube-free crucial words over $\mathcal{A}_{n}$ for $n=1,2,3,4$ of lengths $2,5,11$, and 20, respectively: 
A construction giving the best possible upper bound for $n \geq 5$ can be easily described by examples, and we do this below (for $n=4,5,6,7$; the construction does not work for $n \leq 3$ ). We also provide a general description. The pattern in the construction is easy to recognize.

\section{An almost optimal construction for crucial abelian cube-free words.}

An almost optimal construction of a crucial abelian cube-free word $W_{n}$ over $\mathcal{A}_{n}$ for $n=4,5,6,7$ is shown below. We use spaces to separate the blocks $\Omega_{n, 1}, \Omega_{n, 2}$, and $\Omega_{n, 3}^{\prime}$ in $W_{n}=\Delta_{n}$.

$$
\begin{gathered}
W_{4}=34423312243322143232122334 \\
W_{5}=45534423312254433221543243212233445 \\
W_{6}=56645534423312265544332216543254321223344556 \\
W_{7}=67756645534423312276655443322176543265432122334455667
\end{gathered}
$$

In general, the block $\Omega_{n, 1}$ in $W_{n}=\Delta_{n}=\Omega_{n, 1} \Omega_{n, 2} \Omega_{n, 3}^{\prime}$ is built by adjoining the factors $i(i+1)(i+1)$ for $i=n-1, n-2, \ldots, 1$. The block $\Omega_{n, 2}$ is built by adjoining together the following factors: $n$, $x x$ for $n-1 \geq x \geq 2$, and $n(n-1) \ldots 2$. Finally, the block $\Omega_{n, 3}^{\prime}$ is built by adjoining the factors $(n-1)(n-2) \ldots 1, x x$ for $2 \leq x \leq n-1$, and finally $n$.

It is easy to see that $\left|W_{n}\right|=9 n-10$. We omit the details of showing that $W_{n}$ is crucial with respect to abelian cubes since this can be shown in a similar manner for the optimal construction described below (see the proof of Theorem 11].

\section{An optimal construction for crucial abelian cube-free words.}

An optimal construction of a crucial abelian cube-free word $E_{n}$ over $\mathcal{A}_{n}$ for $n=4,5,6,7$ works as follows. As above, we use spaces to separate the blocks $\Omega_{n, 1}, \Omega_{n, 2}$, and $\Omega_{n, 3}^{\prime}$ in $E_{n}=\Delta_{n}$.

$$
\begin{gathered}
E_{4}=34423311342311343233411 \\
E_{5}=45534423311453423113454323344511 \\
E_{6}=56645534423311564534231134565432334455611 \\
E_{7}=67756645534423311675645342311345676543233445566711
\end{gathered}
$$

In general, the block $\Omega_{n, 1}$ in $E_{n}=\Delta_{n}=\Omega_{n, 1} \Omega_{n, 2} \Omega_{n, 3}^{\prime}$ is built by adjoining the factors $i(i+1)(i+1)$ for $i=n-1, n-2, \ldots, 2$, followed by two 1's. The block $\Omega_{n, 2}$ is built by adjoining the following factors: $i(i+1)$ for $i=n-1, n-2, \ldots, 2$, followed by 11 , and then the factor $34 \cdots(n-1) n$. Finally, the block $\Omega_{n, 3}^{\prime}$ is built by adjoining the factors $(n-1)(n-2) \cdots 32$, then $x x$ for $3 \leq x \leq n-1$, followed by $n$, and finally two 1 's.

We have $E_{n}=\Omega_{n, 1} \Omega_{n, 2} \Omega_{n, 3}^{\prime}$ where $\Omega_{n, 3}=\Omega_{n, 3}^{\prime} n$, and by construction each $\Omega_{n, i}$ contains two 1's, one 2, two $n$ 's, and three $x$ 's for $x=3, \ldots, n-1$. That is, for each $i=1,2,3$, the Parikh vector of the block $\Omega_{n, i}$ is given by $\mathcal{P}\left(\Omega_{n, i}\right)=(2,1,3,3, \ldots, 3,2)$. Hence, $\mathcal{P}\left(E_{n}\right)=(6,3,9,9, \ldots, 9,5)$, and therefore $\left|E_{n}\right|=6+3+9(n-3)+5=9 n-13$. Moreover, for all $n \geq 4$, the word $E_{n}$ is crucial with respect to abelian cubes. We omit the proof of this latter fact since it is very similar to the proof of Theorem 11 (to follow), from which the fact can actually be deduced by setting $k=3$ (in view of Remark 3 , later). Thus, a minimal crucial word avoiding abelian cubes has length at most $9 n-13$ for $n \geq 4$. That is: 
Theorem 2 For $n \geq 4$, we have $\ell_{3}(n) \leq 9 n-13$.

Proof: See the proof of Theorem 11 where one needs to set $k=3$ (in view of Remark 3 , later).

\subsection{A lower bound for $\ell_{3}(n)$}

If $X=\Delta_{n}$ is a crucial word over $\mathcal{A}_{n}$ with respect to abelian cubes, then clearly the number of occurrences of each letter except $n$ must be divisible by 3 , whereas the number of occurrences of $n$ is 2 modulo 3 . We sort in non-decreasing order the number of occurrences of the letters $1,2, \ldots, n-1$ in $X$ to get a nondecreasing sequence of numbers $\left(a_{1} \leq a_{2} \leq \cdots \leq a_{n-1}\right)$. Notice that $a_{i}$ does not necessarily correspond to the letter $i$. We denote by $a_{0}$ the number of occurrences of the letter $n$. Also note that $a_{0}$ can be either larger or smaller than $a_{1}$. By definitions, $|X|=\sum_{i=0}^{n-1} a_{i}$.

For example, the abelian cube-free crucial word $E_{n}$ of length $9 n-13$ in Sec. 2.1 has the following sequence of $a_{i}$ 's: $\left(a_{0}, a_{1}, \ldots, a_{n-1}\right)=(5,3,6,9, \ldots, 9)$. In this subsection, we prove that this sequence cannot be improved for $n \geq 5$, meaning that, e.g., 5 cannot be replaced by 2 , and/or 6 cannot be replaced by 3 , and/or 9('s) cannot be replaced by 3('s) or 6('s), no matter what construction we use to form a crucial word. This is a direct consequence of Lemmas 47 (below) and is recorded in Theorem 8 . In the rest of this section we use, without explanation, the following two facts that are easy to see from the definitions. For any letter $x$ in a crucial abelian cube-free word $X$ over $\mathcal{A}_{n}$ :

1. $\left|\Delta_{x}\right|_{x} \equiv 2(\bmod 3)$ and $\left|\Delta_{x}\right|_{y} \equiv 0(\bmod 3)$ for any other letter $y$ occurring in $X$.

2. If $x+1$ occurs in $X$, then we have $\Delta_{x+1}=Y_{x+1} \Delta_{x}$ where $\left|Y_{x+1}\right|_{x+1} \equiv 2(\bmod 3),\left|Y_{x+1}\right|_{x} \equiv 1$ $(\bmod 3)$, and $\left|Y_{x+1}\right|_{y} \equiv 0(\bmod 3)$ for any other letter $y$ occurring in $X$.

The following fact will also be useful.

Lemma 3 Suppose $X$ is a crucial abelian cube-free word over $\mathcal{A}_{n}$ containing letters $x$ and $y$ such that $x<y<n$ and $|X|_{x}=|X|_{y}=6$. Then $\Delta_{x}$ cannot contain 5 occurrences of the letter $x$.

Proof: Suppose to the contrary that (under the hypotheses of the lemma) $\Delta_{x}$ contains 5 occurrences of the letter $x$. Let $A_{1}=Y_{n} Y_{n-1} \cdots Y_{y+1}$ and $A_{2}=Y_{y} Y_{y-1} \cdots Y_{x+1}$ so that $X=A_{1} A_{2} \Delta_{x}$. Then $\left|A_{1} A_{2}\right|_{x}=1$ and $\left|A_{1} A_{2}\right|_{y} \geq 3$, contradicting the fact that each of the blocks $\Omega_{n, 1}, \Omega_{n, 2}$, and $\Omega_{n, 3}^{\prime}$ in $X=\Delta_{n}=\Omega_{n, 1} \Omega_{n, 2} \Omega_{n, 3}^{\prime}$ must each receive two $x$ 's and two $y$ 's.

Lemma 4 For a crucial abelian cube-free word $X$ over $\mathcal{A}_{n}$, the sequence of $a_{i}$ 's cannot contain 3,3 . That is, $\left(a_{1}, a_{2}\right) \neq(3,3)$.

Proof: Suppose that $x$ and $y$ are letters such that $x<y<n$ and $|X|_{x}=|X|_{y}=3$. Let $A_{1}=$ $Y_{n} Y_{n-1} \cdots Y_{y+1}$ and $A_{2}=Y_{y} Y_{y-1} \cdots Y_{x+1}$ so that we have $X=A_{1} A_{2} \Delta_{x}$. Then we must have the following distribution of $x$ 's and $y$ 's in $X:\left|A_{1}\right|_{y}=1,\left|A_{2}\right|_{y}=2,\left|A_{2}\right|_{x}=1$, and $\left|\Delta_{x}\right|_{x}=2$. However, we get a contradiction, since each of the blocks $\Omega_{n, 2}$ and $\Omega_{n, 3}^{\prime}$ in $X=\Delta_{n}=\Omega_{n, 1} \Omega_{n, 2} \Omega_{n, 3}^{\prime}$ must receive one copy of $x$ and one copy of $y$, which is impossible (no $x$ can exist between the two rightmost $y$ 's).

Lemma 5 For a crucial abelian cube-free word $X$ over $\mathcal{A}_{n}$, the sequence of $a_{i}$ 's cannot contain $6,6,6$. 
Proof: Suppose that $x, y, z$ are three letters such that $x<y<z<n$ and $|X|_{x}=|X|_{y}=|X|_{z}=6$. Let $A_{1}=Y_{n} Y_{n-1} \cdots Y_{z+1}, A_{2}=Y_{z} Y_{z-1} \cdots Y_{y+1}$, and $A_{3}=Y_{y} Y_{y-1} \cdots Y_{x+1}$ so that $X=A_{1} A_{2} A_{3} \Delta_{x}$. Then the minimal requirements on the $A_{i}$ are as follows: $\left|A_{1}\right|_{z} \geq 1,\left|A_{2}\right|_{z} \geq 2,\left|A_{2}\right|_{y} \geq 1,\left|A_{3}\right|_{y} \geq 2$, and $\left|A_{3}\right|_{x} \geq 1$. Moreover, applying Lemma 3 to $x$ and $y$, we have $\left|\Delta_{x}\right|_{2}=2$. And applying the same lemma to the letters $y$ and $z$ guarantees that $A_{1} A_{2}$ contains $4 y$ 's (in particular, $\Delta_{x}$ does not contain any $y$ 's).

Looking at $X=\Delta_{n}=\Omega_{n, 1} \Omega_{n, 2} \Omega_{n, 3}^{\prime}$, we see that for each $i=1,2,3,\left|\Omega_{n, i}\right|_{x}=\left|\Omega_{n, i}\right|_{y}=\left|\Omega_{n, i}\right|_{z}=$ 2. Thus, in $A_{3}$, we must have the following order of letters: $x, y, y$ and the boundary between $\Omega_{n, 2}$ and $\Omega_{n, 3}^{\prime}$ must be between $x$ and $y$ in $A_{3}$. But then $\Delta_{x}$ entirely belongs to $\Omega_{n, 3}^{\prime}$, so it cannot contain any $z$ 's (if it would do so, $\Delta_{x}$ would then contain $3 z$ 's which is impossible). On the other hand, we must have $\left|A_{3}\right|_{z}=3$ for $\Omega_{n, 3}^{\prime}$ to receive $2 z$ 's. Thus, $\Delta_{y}$ contains $2 y$ 's, $3 z$ 's, and $3 x$ 's, which is impossible by Lemma 4 applied to the word $\Delta_{y}$ with two letters occurring exactly 3 times each. (Alternatively, one can see, due to the considerations above, that no $z$ can be between the two rightmost $x$ 's, contradicting the structure of $\Delta_{y}$ ).

Lemma 6 For a crucial abelian cube-free word $X$ over $\mathcal{A}_{n}$, the sequence of $a_{i}$ 's cannot contain $3,6,6$.

Proof: Suppose that $x, y$, and $z$ are letters such that $|X|_{x}=3$ and $|X|_{y}=|X|_{z}=6$. We consider three cases covering all the possibilities up to renaming $y$ and $z$.

Case 1: $z<y<x<n$. One can see that $\Delta_{y}$ does not contain $x$, but it contains at least $3 z$ 's contradicting the fact that each of the blocks $\Omega_{n, 1}, \Omega_{n, 2}$, and $\Omega_{n, 3}^{\prime}$ must receive $1 x$ and $2 z$ 's.

Case 2: $x<z<y<n$. We let $A=Y_{n} Y_{n-1} \cdots Y_{z+1}$ so that $X=A \Delta_{z}$. All three $x$ 's must be in $\Delta_{z}$, while $A$ must contain at least $3 y$ 's contradicting the fact that each of the blocks $\Omega_{n, 1}, \Omega_{n, 2}$, and $\Omega_{n, 3}^{\prime}$ must receive $1 x$ and $2 y$ 's.

Case 3: $z<x<y<n$. We let $A_{1}=Y_{n} Y_{n-1} \cdots Y_{y+1}, A_{2}=Y_{y} Y_{y-1} \cdots Y_{x+1}$, and $A_{3}=$ $Y_{x} Y_{x-1} \cdots Y_{z+1}$ so that $X=A_{1} A_{2} A_{3} \Delta_{z}$. The minimal requirements on the $A_{i}$ and $\Delta_{z}$ are as follows: $\left|A_{1}\right|_{y} \geq 1,\left|A_{2}\right|_{y} \geq 2,\left|A_{2}\right|_{x}=1,\left|A_{3}\right|_{x}=2,\left|A_{3}\right|_{z} \geq 1$, and $\left|\Delta_{z}\right|_{z} \geq 2$. Now $\Delta_{z}$ cannot contain $3 y$ 's, for otherwise, considering the structure of $\Delta_{x}$, it would not be possible to distribute $x$ 's and $y$ 's in a proper way. However, if $A_{3}$ contains $3 y$ 's then, so as not to contradict the structure of $\Delta_{x}$ (no proper distribution of $y$ 's and $z$ 's would exist), $\Delta_{x}$ must contain $3 z$ 's, which contradicts to the structure of $X=\Delta_{n}=\Omega_{n, 1} \Omega_{n, 2} \Omega_{n, 3}^{\prime}$ (no proper distribution of $y$ 's and $z$ 's would exist among the blocks $\Omega_{n, 1}, \Omega_{n, 2}$, and $\Omega_{n, 3}^{\prime}$, each of which is supposed to contain exactly 2 occurrences of $y$ and 2 occurrences of $z$ ). Thus, there are no $y$ 's in $\Delta_{x}$, contradicting the structure of $\Delta_{n}$ (no proper distribution of $y$ 's and $x$ 's would exist among the blocks $\Omega_{n, 1}, \Omega_{n, 2}$, and $\Omega_{n, 3}^{\prime}$ ).

Lemma 7 For a crucial abelian cube-free word $X$ over $\mathcal{A}_{n}$,

$$
\left(a_{0}, a_{1}, a_{2}, a_{3}, a_{4}\right) \neq(2,3,6,9,9) .
$$


Proof: Suppose $|X|_{n}=2$ and assume that $|X|_{t}=3$ for some other letter $t$. If $t \neq n-1$, then all three occurrences of $t$ are in $\Delta_{n-1}$, whereas the two occurrences of $n$ are in $Y_{n}$ (recall that $X=\Delta_{n}=$ $\left.Y_{n} \Delta_{n-1}\right)$. This contradicts the fact that $\left|\Omega_{n, 1}\right|_{n}=\left|\Omega_{n, 1}\right|_{t}=1$. Thus, $t=n-1$ and $|X|_{n-1}=3$.

Now, assuming $x, y$, and $z$ are three letters, with $x<y<z<n-1$, occurring $\{6,9,9\}$ times in $X$ (we do not specify which letter occurs how many times). Then, as in the proof of Lemma 5, we deduce that $\Delta_{z}$ belongs entirely to the block $\Omega_{n, 3}^{\prime}$. Moreover, the block $\Omega_{n, 3}^{\prime}$ has $\{2,3,3\}$ occurrences of letters $x, y, z$ (in some order). However, if $x$ or $y$ occur twice in $\Omega_{n, 3}^{\prime}$, they occur twice in $\Delta_{z}$, contradicting the structure of $\Delta_{z}$. Thus $z$ must occur twice in $\Omega_{n, 3}^{\prime}$, and the letters $x$ and $y$ each occur 3 times in $\Omega_{n, 3}^{\prime}$. But then it is clear that $x$ and $y$ must each occur 3 times in $\Delta_{z}$, contradicting the fact that $x$ and $z$ should be distributed properly in $\Delta_{z}$, by Lemma 4 .

Theorem 8 For $n \geq 5$, we have $\ell_{3}(n) \geq 9 n-13$.

Proof: This is a direct consequence of the preceding four lemmas, which tell us that any attempt to decrease numbers in the sequence $(5,3,6,9,9, \ldots, 9)$ corresponding to $E_{n}$ will lead to a prohibited configuration.

Corollary 9 For $n \geq 5$, we have $\ell_{3}(n)=9 n-13$.

Proof: The result follows immediately from Theorems 2 and 8

Remark 1 Recall from Sec. 2.1 that $\ell_{3}(n)=2,5,11,20$ for $n=1,2,3,4$, respectively. For instance, the word 42131214231211321211 is a minimal crucial abelian cube-free word over $\mathcal{A}_{4}$ of length 20 $(=2+3+6+9)$. This can be proved using similar arguments as in the proofs of the Lemmas 47

\section{Crucial words for abelian $k$-th powers}

\subsection{An upper bound for $\ell_{k}(n)$ and a conjecture}

The following theorem is a direct generalization of Theorem 1 and is a natural approach to obtaining an upper bound that improves $k^{n}-1$ given by the $k$-generalized Zimin word $Z_{n, k}$.

Theorem 10 For $k \geq 3$, we have $\ell_{k}(n) \leq k \cdot(k-1)^{n-1}-1$.

Proof: We proceed as in the proof of Theorem 11, with the only difference being that we begin with $X_{1}=1^{k-1}$ and put $(k-2) 1$ 's to the right of each letter except for the last $(k-2)$ letters, after which we put $(k-1) 1$ 's instead.

We skip here the analysis of the work of a greedy algorithm, and proceed directly with the construction of a crucial abelian $k$-power-free word $W_{n, k}$ over $\mathcal{A}_{n}$ before describing the construction of a similar word $D_{n, k}$ that we believe to be optimal.

A construction of a crucial abelian $k$-power-free word $W_{n, k}$, where $n \geq 4$ and $k \geq 3$.

We illustrate each step of the algorithm by example, letting $k=n=4$. 
The construction can be explained directly, but we introduce it recursively, obtaining, for $n \geq 4, W_{n, k}$ from $W_{n, k-1}$ and using the abelian cube case $W_{n, 3}=W_{n}$ as the basis. For $n=4$,

$$
W_{4,3}=\Omega_{4,1} \Omega_{4,2} \Omega_{4,3}^{\prime}=34423312243322143232122334 .
$$

Assume that $W_{n, k-1}=\Omega_{n, 1} \Omega_{n, 2} \ldots \Omega_{n, k-1}^{\prime}$ is constructed and implement the following steps to obtain $W_{n, k}$ :

1. Duplicate $\Omega_{n, 1}$ in $W_{n, k-1}$ to obtain the word

$$
W_{n, k-1}^{\prime}=\Omega_{n, 1} \Omega_{n, 1} \Omega_{n, 2} \ldots \Omega_{n, k-1}^{\prime} .
$$

For $n=k=4$,

$$
W_{4,3}^{\prime}=34423312234423312243322143232122334 .
$$

2. Append to the second $\Omega_{n, 1}$ in $W_{n, k}^{\prime}$ the factor $n(n-1) \ldots 2$ (in our example, 432) to obtain $\Omega_{n, 2}$ in $W_{n, k}$, and in each of the remaining blocks $\Omega_{n, i}$ in $W_{n, k-1}^{\prime}$, duplicate the rightmost occurrence of each letter $x$, where $2 \leq x \leq n$, to obtain $W_{n, k}$. For $n=k=4$,

$$
W_{4,4}=34442333122234423312243243322144332232122233344 .
$$

We provide two more examples here, namely $W_{5,4}$ and $W_{4,5}$, respectively, so that readers can check their understanding of the construction:

$$
455534442333122245534423312254325443322155443322432122233344455 ;
$$

34444233331222234442333122243234423312244332243322144433322232122223333444.

It is easy to see that $\left|W_{n, k}\right|=k^{2}(n-1)-1$. We omit the proof that $W_{n, k}$ is crucial with respect to abelian $k$-th powers since it is similar to the proof for the following word $D_{n, k}$, which is $k$ letters shorter than $W_{n, k}$ (see Theorem 11).

A construction of a crucial abelian $k$-power-free word $D_{n, k}$, where $n \geq 4$ and $k \geq 2$.

As we shall see, the following construction of the word $D_{n, k}$ is optimal for $k=2,3$. We believe that it is also optimal for any $k \geq 4$ and sufficiently large $n$ (see Conjecture 1).

As our basis for the construction of the word $D_{n, k}$, we use the following word $D_{n}$, which is constructed as follows, for $n=4,5,6,7$. (As previously, we use spaces to separate the blocks $\Omega_{n, 1}$ and $\Omega_{n, 2}^{\prime}$ in $D_{n}=\Delta_{n}$.)

$$
\begin{gathered}
D_{4}=342313231 \\
D_{5}=4534231432341 \\
D_{6}=56453423154323451 \\
D_{7}=675645342316543234561
\end{gathered}
$$

In general, the first block $\Omega_{n, 1}$ in $D_{n}=\Delta_{n}=\Omega_{n, 1} \Omega_{n, 2}^{\prime}$ is built by adjoining the factors $i(i+1)$ for $i=n-1, n-2, \ldots, 2$, followed by the letter 1 . The second block $\Omega_{n, 2}^{\prime}$ is built by adjoining the factors $(n-1)(n-2) \cdots 432$, then $34 \cdots(n-2)(n-1)$, and finally the letter 1 . 
Remark 2 The above construction coincides with the construction given in [6] Theorem 5] for a minimal crucial abelian square-free word over $\mathcal{A}_{n}$ of length $4 n-7$. In fact, the word $D_{n}$ can be obtained from the minimal crucial abelian cube-free word $E_{n}$ (defined in Sec. 2.1) by removing the second block in $E_{n}$ and deleting the rightmost copy of each letter except 2 in the first and third blocks of $E_{n}$.

Now we illustrate each step of the construction for the word $D_{n, k}$ by example, letting $n=4$ and $k=3$. The construction is very similar to that of $W_{n, k}$ and can be explained directly, but we introduce it recursively, obtaining $D_{n, k}$ from $D_{n, k-1}$ for $n \geq 4$, and using the crucial abelian square-free word $D_{n, 2}:=D_{n}$ as the basis. For $n=4$, we have

$$
D_{4,2}=\Omega_{4,1} \Omega_{4,2}^{\prime}=342313231 .
$$

Assume that $D_{n, k-1}=\Omega_{n, 1} \Omega_{n, 2} \cdots \Omega_{n, k-1}^{\prime}$ is constructed and implement the following steps to obtain $D_{n, k}$ :

1. Duplicate $\Omega_{n, 1}$ in $D_{n, k-1}$ to obtain the word

$$
D_{n, k-1}^{\prime}=\Omega_{n, 1} \Omega_{n, 1} \Omega_{n, 2} \cdots \Omega_{n, k-1}^{\prime} .
$$

For $n=4$ and $k=3, D_{4,2}^{\prime}=34231342313231$.

2. Append to the second $\Omega_{n, 1}$ in $D_{n, k-1}^{\prime}$ the factor $134 \cdots n$ (in our example, 134; in fact, any permutation of $\{1,3,4, \ldots, n\}$ would work at this place) to obtain $\Omega_{n, 2}$ in $D_{n, k}$. In each of the remaining blocks $\Omega_{n, i}$ in $D_{n, k-1}^{\prime}$, duplicate the rightmost occurrence of each letter $x$, where $1 \leq x \leq n$ and $x \neq 2$. Finally, in the last block of $D_{n, k}^{\prime}$ insert the letter $n$ immediately before the leftmost 1 to obtain the word $D_{n, k}$. For $n=4$ and $k=3$, we have

$$
D_{4,3}=34423311342311343233411
$$

where the bold letters form the word $D_{4,2}^{\prime}$ from which $D_{4,3}$ is derived.

We provide five more examples here, namely $D_{5,3}, D_{5,4}, D_{4,4}, D_{4,5}$, and $D_{6,4}$, respectively, so that readers can check their understanding of the construction:

$$
45534423311453423113454323344511 \text {; }
$$

45553444233311145534423311134545342311133445543233344455111 ;

3444233311134423311134342311133443233344111

344442333311113444233311113434423311113344342311113334443233334441111 ;

566645553444233311156645534423311134565645342311133445566543233344455566111.

Remark 3 By construction, $D_{n, 3}=E_{n}$ for all $n \geq 4$.

Theorem 11 For $n \geq 4$ and $k \geq 2$, we have $\ell_{k}(n) \leq k^{2}(n-1)-k-1$. 
Proof: Fix $n \geq 4$ and $k \geq 2$. We have

$$
D_{n, k}=\Omega_{n, 1} \Omega_{n, 2} \cdots \Omega_{n, k-1} \Omega_{n, k}^{\prime}
$$

where $\Omega_{n, k}=\Omega_{n, k}^{\prime} n$, and by construction each $\Omega_{n, i}$ contains $(k-1)$ occurrences of the letter 1 , one occurrence of the letter $2,(k-1)$ occurrences of the letter $n$, and $k$ occurrences of the letter $x$ for $x=3,4, \ldots, n-1$. That is, for each $i=1,2, \ldots, k$, the Parikh vector of the block $\Omega_{n, i}$ is given by

$$
\mathcal{P}\left(\Omega_{n, i}\right)=(k-1,1, k, k, \ldots, k, k-1),
$$

and hence $\mathcal{P}\left(D_{n, k}\right)=\left(k(k-1), k, k^{2}, k^{2}, \ldots, k^{2}, k(k-1)-1\right)$. Thus,

$$
\left|D_{n, k}\right|=k(k-1)+k+k^{2}(n-3)+k(k-1)-1=k^{2}(n-1)-k-1 .
$$

We will now prove that $D_{n, k}$ is crucial with respect to abelian $k$-th powers; whence the result. The following facts, which are easily verified from the construction of $D_{n, k}$, will be useful in the proof.

Fact 1. In every block $\Omega_{n, i}$, the letter 3 has occurrences before and after the single occurrence of the letter 2 .

Fact 2. In every block $\Omega_{n, i}$, all $(k-1)$ of the 1 's occur after the single occurrence of the letter 2 (as the factor $1^{k-1}$ ).

Fact 3. For all $i$ with $2 \leq i \leq k-1$, the block $\Omega_{n, i}$ ends with $n^{i-1}$ and the other $(k-1-i+1) n$ 's occur (together as a string) before the single occurrence of the letter 2 in $\Omega_{n, i}$. In particular, there are exactly $k-2$ occurrences of the letter $n$ between successive 2 's in $D_{n, k}$.

Freeness: First we prove that $D_{n, k}$ is abelian $k$-power-free. Obviously, by construction, $D_{n, k}$ is not an abelian $k$-th power (as the number of occurrences of the letter $n$ is not a multiple of $k$ ) and $D_{n, k}$ does not contain any trivial $k$-th powers, i.e., $k$-th powers of the form $x^{k}$ for some letter $x$. Moreover, each block $\Omega_{n, i}$ is abelian $k$-power-free. For if not, then according to the Parikh vector of $\Omega_{n, i}$ (see (1)), at least one of the $\Omega_{n, i}$ must contain an abelian $k$-th power consisting of exactly $k$ occurrences of the letter $x$ for all $x=3,4, \ldots, n-1$, and no occurrences of the letters 1,2 , and $n$. But, by construction, this is impossible because, for instance, the letter 3 has occurrences before and after the letter 2 in each of the blocks $\Omega_{n, i}$ in $D_{n, k}$ (by Fact 1).

Now suppose, by way of contradiction, that $D_{n, k}$ contains a non-trivial abelian $k$-th power, say $P$. Then it follows from the preceding paragraph that $P$ overlaps at least two of the blocks $\Omega_{n, i}$ in $D_{n, k}$. We first show that $P$ cannot overlap three or more of the blocks in $D_{n, k}$. For if so, then $P$ must contain at least one of the blocks, and hence $P$ must also contain all $k$ of the 2 's. Furthermore, all of the 1 's in each block occur after the letter 2 (by Fact 2), so there are $(k-1)^{2}=k^{2}-2 k+1$ occurrences of the letter 1 between the leftmost and rightmost 2's in $D_{n, k}$. Thus, $P$ must contain all $k(k-1)=k^{2}-k$ of the 1 's. Hence, since $\Omega_{n, k}^{\prime}$ ends with $1^{k-1}$, we deduce that $P$ must end with the word

$$
W=23^{k-1} 1^{k-1} \Omega_{n, 2} \cdots \Omega_{n, k-1} \Omega_{n, k}^{\prime},
$$

where $|W|_{n}=k,|W|_{3}=k(k-1)+(k-1)=k^{2}-1$, and $|W|_{x}=k(k-1)$ for $x=4, \ldots, n-1$. It follows that $P$ must contain all $k^{2}$ of the 3 's. But then, since

$$
D_{n, k}=(n-1) n^{k-1} \cdots 34^{k-1} W
$$


(by construction), we deduce that $P$ must contain all $k^{2}$ of the 4 's that occur in $D_{n, k}$, and hence all $k^{2}$ of the 5's, and so on. That is, $P$ must contain all $k^{2}$ occurrences of the letter $x$ for $x=3, \ldots, n-1$; whence, since $D_{n, k}$ begins with the letter $n-1$, we have $P=\Omega_{n, 1} \Omega_{n, 2} \cdots \Omega_{n, k}=D_{n, k}$, a contradiction.

Thus, $P$ overlaps exactly two adjacent blocks in $D_{n, k}$, in which case $P$ cannot contain the letter 2 ; otherwise $P$ would contain all $k$ of the 2 's, and hence would overlap all of the blocks in $D_{n, k}$, which is impossible (by the preceding arguments). Hence, $P$ lies strictly between two successive occurrences of the letter 2 in $D_{n, k}$. But then $P$ cannot contain the letter $n$ as there are exactly $k-2$ occurrences of the letter $n$ between successive 2's in $D_{n, k}$ (by Fact 3 ). Therefore, since the blocks $\Omega_{n, i}$ with $2 \leq i \leq k-1$ end with the letter $n$, it follows that $P$ overlaps the blocks $\Omega_{n, 1}$ and $\Omega_{n, 2}$. Now, by construction, $\Omega_{n, 1}$ ends with $1^{k-1}$, and hence $P$ contains $k$ of the $2(k-1)=2 k-2$ occurrences of the letter 1 in $\Omega_{n, 1} \Omega_{n, 2}$. But then $P$ must contain the letter 2 because $\Omega_{n, 1}$ contains exactly $(k-1)$ occurrences of the letter 1 (as a suffix) and all $(k-1)$ of the 1's in $\Omega_{n, 2}$ occur after the letter 2 (by Fact 2); a contradiction.

We have now shown that $D_{n, k}$ is abelian $k$-power-free. It remains to show that $D_{n, k} x$ ends with an abelian $k$-th power for each letter $x=1,2, \ldots, n$.

Cruciality: By construction, $D_{n, k} n$ is clearly an abelian $k$-th power. It is also easy to see that $D_{n, k} 1$ ends with the (abelian) $k$-th power $\Delta_{1} 1:=1^{k}$. Furthermore, for all $m=n, n-1, \ldots, 4$, we deduce from the construction that

$$
\begin{aligned}
\Omega_{m, 1} & =(m-1) m^{k-1} \Omega_{m-1,1}, \\
\Omega_{m, 2} & =(m-1) m^{k-2} \Omega_{m-1,2} m, \\
& \vdots \\
\Omega_{m, k-2} & =(m-1) m^{2} \Omega_{m-1, k-2} m^{k-3}, \\
\Omega_{m, k-1} & =(m-1) m \Omega_{m-1, k-1} m^{k-2}, \\
\Omega_{m, k}^{\prime} & =(m-1) \Omega_{m-1, k}^{\prime}\left[1^{k-1}\right]^{-1}(m-1) m^{k-2} 1^{k-1},
\end{aligned}
$$

where $\Omega_{m-1, k}^{\prime}\left[1^{k-1}\right]^{-1}$ indicates the deletion of the suffix $1^{k-1}$ of $\Omega_{m-1, k}^{\prime}$.

Consequently, for $x=n-1, n-2, \ldots, 3$, the word $D_{n, k} x$ ends with the abelian $k$-th power $\Delta_{x} x$ where $\Delta_{x}$ is such that

$$
\Delta_{x+1}=x(x+1)^{k-1} \Delta_{x} \quad \text { with } \Delta_{n}:=D_{n, k} .
$$

Observe that $\left|D_{n, 2}\right|=4 n-7$ and $\left|D_{n, 3}\right|=9 n-13$. Hence, since $D_{n, k}$ is a crucial abelian $k$-power-free word (by the proof of Theorem 11], it follows from [6, Theorem 5] and Corollary 9 that the words $D_{n, 2}$ and $D_{n, 3}$ are minimal crucial words over $\mathcal{A}_{n}$ avoiding abelian squares and abelian cubes, respectively. That is, for $k=2,3$, the word $D_{n, k}$ gives the length of a minimal crucial word over $\mathcal{A}_{n}$ avoiding abelian $k$-th powers. In the case of $k \geq 4$, we make the following conjecture.

Conjecture 1 For $k \geq 4$ and sufficiently large n, the length of a minimal crucial word over $\mathcal{A}_{n}$ avoiding abelian $k$-th powers is given by $k^{2}(n-1)-k-1$. 


\subsection{A Lower Bound for $\ell_{k}(n)$}

A trivial lower bound for $\ell_{k}(n)$ is $n k-1$ as all letters except $n$ must occur at least $k$ times, whereas $n$ must occur at least $k-1$ times. We give here the following slight improvement of the trivial lower bound, which must be rather imprecise though.

Theorem 12 For $n \geq 5$ and $k \geq 4$, we have $\ell_{k}(n) \geq k(3 n-4)-1$.

Proof: Assuming that $X$ is a crucial word over the $n$-letter alphabet $\mathcal{A}_{n}$ with respect to abelian $k$-th powers $(k \geq 4)$, we see that adjoining any letter from $\mathcal{A}_{n}$ to the right of $X$ must create a cube as a factor from the right. In particular, adjoining $n$ from the right side leads to creating a cube of length at least $9 n-13$ (by Lemmas 4 77. This cube will be $\Omega_{n, k-2} \Omega_{n, k-1} \Omega_{n, k}^{\prime}$ in $X$ and thus $\Omega_{n, i}$, for $1 \leq i \leq k-1$, will have length at least $3 n-4$, whereas $\Omega_{n, k}^{\prime}$ has length at least $3 n-5$, which yields the result.

\section{Further Research}

1. Prove or disprove Conjecture 1. Notice that the general construction uses a greedy algorithm for going from $k-1$ to $k$, which does not work for going from $n-1$ to $n$ for a fixed $k$. However, we believe that the conjecture is true.

2. A word $W$ over $\mathcal{A}_{n}$ is maximal with respect to a given set of prohibitions if $W$ avoids the prohibitions, but $x W$ and $W x$ do not avoid the prohibitions for any letter $x \in \mathcal{A}_{n}$. For example, the word 323121 is a maximal abelian square-free word over $\{1,2,3\}$ of minimal length. Clearly, the length of a minimal crucial word with respect to a given set of prohibitions is at most the length of a shortest maximal word. Thus, obtaining the length of a minimal crucial word we get a lower bound for the length of a shortest maximal word.

Can we use our approach to tackle the problem of finding maximal words of minimal length? In particular, Korn [8] proved that the length $\ell(n)$ of a shortest maximal abelian square-free word over $\mathcal{A}_{n}$ satisfies $4 n-7 \leq \ell(n) \leq 6 n-10$ for $n \geq 6$, while Bullock [1] refined Korn's methods to show that $6 n-29 \leq \ell(n) \leq 6 n-12$ for $n \geq 8$. Can our approach improve Bullock's result (probably too much to ask when taking into account how small the gap is), or can it provide an alternative solution?

\section{Acknowledgements}

The authors would like to thank an anonymous referee for helpful comments and suggestions. The first and third authors were supported by the Icelandic Research Fund (grant nos. 09003801/1 and 060005012/3). 


\section{References}

[1] E.M. Bullock, Improved bounds on the length of maximal abelian square-free words, Electron. J. Combin. 11 (2004) \#R17.

[2] A. Carpi, On the number of abelian square-free words on four letters, Discrete Appl. Math. 81 (1998) 155-167.

[3] L.J. Cummings, M. Mays, A one-sided Zimin construction, Electron. J. Combin. 8 (2001), \#R27.

[4] P. Erdős, Some unsolved problems, Magyar Tud. Akad. Mat. Kutató Int. Közl. 6 (1961) 221-254.

[5] A.A. Evdokimov, Strongly asymmetric sequences generated by a finite number of symbols, Dokl. Akad. Nauk SSSR 179 (1968) 1268-1271; Soviet Math. Dokl. 9 (1968) 536-539.

[6] A. Evdokimov, S. Kitaev, Crucial words and the complexity of some extremal problems for sets of prohibited words, J. Combin. Theory Ser. A 105 (2004) 273-289.

[7] V. Keränen, Abelian squares are avoidable on 4 letters, in: Automata, languages and programming (Vienna, 1992), 41-52; Lecture Notes in Comput. Sci., vol. 623, Springer, Berlin, 1992.

[8] M. Korn, Maximal abelian square-free words of short length, J. Combin. Theory Ser. A 102 (2003) 207-211.

[9] P.A.B. Pleasants, Non-repetitive sequences, Proc. Cambridge Philos. Soc. 68 (1970) 267-274.

[10] A. Zimin, Blocking sets of terms, Mat. Sb. (N.S.) 119 (161) (1982) 363-375, 447; Math. USSR Sbornik 47 (1984) 353-364. 